\title{
SINGLE CHANNEL CURRENTS ACTIVATED BY $\gamma$-AMINOBUTYRIC ACID, MUSCIMOL, AND (-)-PENTOBARBITAL IN CULTURED MOUSE SPINAL NEURONS
}

\author{
MEYER B. JACKSON, ${ }^{*, 1}$ HAROLD LECAR, ${ }^{*}$ DAVID A. MATHERS, $\ddagger$ AND JEFFREY L. BARKER \\ ${ }^{*}$ Laboratory of Biophysics and $\ddagger$ Laboratory of Neurophysiology, National Institute of Neurological and Communicative \\ Disorders and Stroke, National Institutes of Health, Bethesda, Maryland 20205
}

Received December 3, 1981; Revised February 5, 1982; Accepted February 22, 1982

\begin{abstract}
The patch electrode technique was used to record single channel current pulses in tissue-cultured mouse spinal cord neurons. In agreement with earlier noise studies, channels activated by $\gamma$ aminobutyric acid (GABA), muscimol, and (-)-pentobarbital were found to have equal unit conductances. The kinetics of channel closing were studied by analyzing the distributions of open state lifetimes. Channels activated by (-)-pentobarbital and muscimol had longer mean open times than channels activated by GABA. As a result, the kinetics of (-)-pentobarbital- and muscimol-activated channels could be studied in greater detail. Most observed open state lifetime distributions were not exponential but contained an excess of short duration events. A sum of two exponential functions gave a much better fit than a single exponential function to most observed open state lifetime distributions. A critical comparison of noise analysis with single channel recording shows that the fast process responsible for the rapid closures would be very difficult to detect in a noise experiment. The channel noise is dominated by the slower process, and as a result, the relaxation time of the slower kinetic component derived from single channel studies is close to the mean open state lifetime derived from noise measurements. The observation of a faster process points toward either an additional population of channels or a scheme for the channel closing transition which is not a simple first order process.
\end{abstract}

The molecular events underlying the generation of postsynaptic currents are thought to be the conformational transitions of chemically gated ionic channels. The first quantitative determination of channel properties in living cell membranes was accomplished by analyzing current fluctuations induced by the application of transmitter to chemically excitable cells (Katz and Miledi, 1970; Anderson and Stevens, 1973; Sachs and Lecar, 1973). The patch electrode technique of Neher and Sakmann (1976) has confirmed and extended noise analysis by allowing the direct observation of single channel currents. In this study, we report single channel currents in mouse spinal cord neurons induced by three agoniststhe putative inhibitory transmitter GABA $(\gamma$-aminobutyric acid), the mushroom toxin muscimol, and the general anesthetic (-)-pentobarbital. In previous work with tissue-cultured mouse spinal cord cells, fluctuation analysis was used to estimate the properties of the chlorideselective channel activated by these substances (Mc-

\footnotetext{
' To whom correspondence should be addressed at his present address: Department of Biology, University of California, 405 Hilgard Avenue, Los Angeles, CA 90024.
}

Burney and Barker, 1978; Mathers and Barker, 1980, 1981b). The electrical noise studies indicate that these substances open chloride ion channels whose conductances are similar but whose durations are significantly different. In particular, corner frequencies of spectra obtained from an analysis of noise induced by muscimol and (-)-pentobarbital are much lower than they are in noise induced by GABA, leading to the supposition that these drugs induce substantially longer channel openings. The single channel measurements provide a direct test of these conclusions and provide additional insight into the conformational transitions of chemically activated channels.

\section{Materials and Methods}

Dissociated spinal cords from 14-day mouse embryos were used to prepare the cell cultures according to techniques previously described (Ransom et al., 1977a). Recordings of channel currents were made by established procedures (Neher and Sakmann, 1976; Jackson and Lecar, 1979; Nelson and Sachs, 1979; Hamill et al., 1981). The cultures were bathed in Hank's saline buffered at pH 7.4 with $20 \mathrm{~mm}$ HEPES. The addition of $10 \mathrm{~mm}$ 
$\mathrm{MgCl}_{2}, 1 \mathrm{mM} \mathrm{CaCl}$, and $0.5 \mu \mathrm{M}$ tetrodotoxin to the bathing medium eliminated spontaneous electrical activity. The recordings were made at room temperature (21 to $23^{\circ} \mathrm{C}$ ) on the stage of an inverted phase contrast microscope. Cells larger than $20 \mu \mathrm{m}$ were impaled with a single intracellular microelectrode ( 40 to 80 megohms) filled with $3 \mathrm{M} \mathrm{KCl}$. Patch electrodes were fabricated in a microforge and had tip inner diameters of approximately $1 \mu \mathrm{m}$. They were filled with solutions identical to the bathing solution but with the addition of either 0.5 to $1.0 \mu \mathrm{M}$ GABA, 0.3 to $1.0 \mu \mathrm{M}$ muscimol, or $50 \mu \mathrm{M}$ (-)-pentobarbital. These concentrations were empirically determined to produce current jumps at a frequency suitable for the analysis of individual channel events. The patch electrodes had resistances of from 2 to 5 megohms after filling and produced seal resistances of 30 to 100 megohms on contact with the surface of the spinal cord cells. Suction was applied to increase seal resistance. These experiments were performed before techniques for obtaining gigohm seals became widely known. Interested readers should consult a review by Hamill et al. (1981) for a description of improved methods of patch electrode recording. A preliminary account of our work was presented at the 1981 Biophysical Society Meeting (Mathers et al., 1981).

The noise level was reduced by passing the signal through a low pass analogue filter. The cutoff frequency, $f_{c}$, was usually $100 \mathrm{~Hz}$, but in some instances, it was varied from 60 to $150 \mathrm{~Hz}$. In filtered records, the efficiency of detecting single channel pulses declines rapidly for pulse durations shorter than $1 /\left(2 \pi f_{c}\right)$. The minimum detectable pulse duration also depends on the noise level. To insure that open state lifetime distributions were not distorted by the bandwidth, only channel current durations greater than or equal to $10 \mathrm{msec}$ were included in the analysis of channel kinetics. Channel currents that were less than $15 \mathrm{msec}$ in duration were not included in current amplitude averages to insure that detectable jumps with attenuated amplitudes did not reduce the estimates of channel conductance.

Resting membrane potentials ranged from -45 to -70 $\mathrm{mV}$. Intracellular injection of chloride ions with a $3 \mathrm{~m}$ $\mathrm{KCl}$-filled microelectrode alters the chloride reversal potential to a more positive value of approximately $-20 \mathrm{mV}$ (Barker and Ransom, 1978). A single intracellular electrode in a bridge circuit was used to record the membrane potential and to hyperpolarize by passing current. The injection of chloride produced an outward chloride driving force with the consequence that channel current pulses were inward as displayed in Figure 1. Nearly every placement of an agonist-filled electrode that produced a high impedance seal resulted in channel events, suggesting that receptors are widely distributed over the surface of the cell soma and not confined exclusively to a few regions of high sensitivity or "hotspots" (Ransom et al., 1977b; Barker and Ransom, 1978). These single channel currents were not seen in control experiments with patch electrodes containing only saline.

\section{Results}

For all compounds used to activate channels, the amplitudes of the current pulses appeared to be distributed in a unimodal fashion. The mean amplitudes of current events evoked by GABA, (-)-pentobarbital, and muscimol at $-80 \mathrm{mV}$ do not differ significantly from each other. This result is in agreement with the analysis of current fluctuations evoked by the three agonists in cells from sister cultures (Mathers and Barker, 1980, 1981b), where the chloride reversal potential measured with $\mathrm{KCl}$ filled electrodes was determined to be approximately -20 $\mathrm{mV}$ (range, -40 to $-10 \mathrm{mV}$ ). Conductances were estimated by dividing the average channel current by the driving force in each experiment. The average channel conductances from all experiments are presented in Table I. These conductances are $30 \%$ to $90 \%$ larger than the average values obtained from noise analysis.

The distribution of current jump durations in records such as those of Figure 1 provides information about the kinetic behavior of channel gating. Figure 2 shows cumulative plots of the number of events, $N(t)$, with durations longer than a specified time, $t$, plotted against $t$. Assuming that an agonist activates a single population of ionic channels having first order kinetics of closing, plots of this kind should be well fit by a single exponential function with a time constant, $\tau$, equal to the mean channel open time. Although the observed distributions varied from one experiment to the next, it is clear that distributions such as those of Figure 2, $B$ and $C$, cannot be adequately described by a single exponential function. For example, a best single exponential fit to the data of Figure $2 B$, would give a value of $\tau=17.7 \mathrm{msec}$, but such a fit would not account for the excess number of very long channel openings. In this particular experiment, 6 of

\section{TABLE I}

Parameters determined from curve fits of one or two exponential functions to distributions such as those presented in Figure 2

The superscripts after the membrane potentials of some of the entries refer to specific curves displayed in Figure 2. The parameters $\tau$ and $N$ refer to the relaxation time and number of events, respectively; the subscripts $F$ and $S$ indicate the fast and slow processes, respectively. The ratio $N_{F} / N_{S}$ is presented since the absolute values of $N_{F}$ and $N_{S}$ are proportional to the total number of events observed in a particular experinent. $\bar{\tau}_{S}$ and $\bar{\tau}_{F}$ were determined for muscinul where more experiments were done.

\begin{tabular}{|c|c|c|c|c|}
\hline & $\begin{array}{c}\text { Membrane } \\
\text { Potential }\end{array}$ & $\tau_{S}$ & $\tau_{F}$ & $N_{H} / N_{S}$ \\
\hline & $m V$ & & & \\
\hline \multirow[t]{6}{*}{ Muscimol } & $-80^{A}$ & $44.7 \pm 7.7$ & $13.7 \pm 7.3$ & 0.69 \\
\hline & $-80^{B}$ & $74.1 \pm 10.4$ & $9.6 \pm 0.6$ & 5.3 \\
\hline & -80 & $52.0 \pm 2.9$ & $7.6 \pm 0.5$ & 3.2 \\
\hline & -70 & $23.7 \pm 1.7$ & $4.9 \pm 6.5$ & 2.4 \\
\hline & -60 & $31.4 \pm 2.2$ & $6.5 \pm 0.9$ & 2.3 \\
\hline & -100 & $43.4 \pm 5.1$ & $7.2 \pm 0.9$ & 3.7 \\
\hline Averages: & & $\bar{\tau}_{S}=45 \pm 17$ & \multicolumn{2}{|l|}{$\bar{\tau}_{F}=8.2 \pm 3.1$} \\
\hline \multicolumn{5}{|c|}{ Conductance $=23.8 \pm 4.2 \mathrm{pS}: 11$ experiments } \\
\hline \multirow[t]{2}{*}{ GABA } & $\begin{array}{l}-80 \\
-80\end{array}$ & \multicolumn{3}{|c|}{$\begin{array}{l}10.0 \pm 0.8 \text { (one exponential fit) } \\
21.5 \pm 0.6 \text { (one exponential fit) }\end{array}$} \\
\hline & $-90^{c}$ & $35.1 \pm 5.3$ & $5.4 \pm 0.4$ & 8.3 \\
\hline \multicolumn{5}{|c|}{ Conductance $=20.7 \pm 3.2 \mathrm{pS}: 7$ experiments } \\
\hline (-)-Pento- & -80 & $71.6 \pm 7.2$ & $11.4 \pm 0.8$ & 4.13 \\
\hline barbital & -80 & $166 \pm 129$ & $24.0 \pm 3.2$ & 9.2 \\
\hline \multicolumn{5}{|c|}{ Conductance $=22.6 \pm 5.8 \mathrm{pS}: 4$ experiments } \\
\hline
\end{tabular}



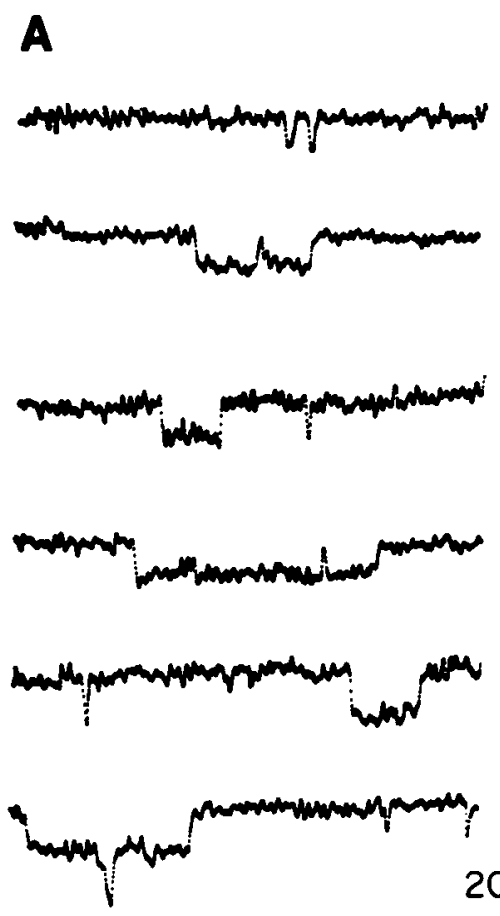

$20 \mathrm{pA}$

$1 \mathrm{sec}$

B
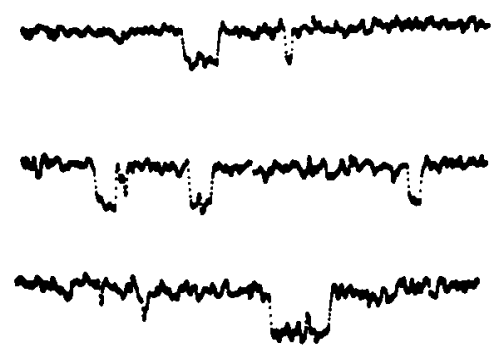

Figure 1. Channel currents recorded from cultured spinal neurons. Currents are recorded on a digital oscilloscope at 1-sec sweeps with a sampling rate of $1024 \mathrm{sec}^{-1} . A$, Channels activated by muscimol. Muscimol records typically show a mixture of short and long jumps as summarized in Table I. The longest event shown in this figure (fourth trace from top) is over 400 msec long. $B$, GABA-induced current jumps. the 185 observed jumps were longer than 200 msec. A $\chi^{2}$ test shows that the probability that the long jumps are chance members of the best fit single exponential population is $10^{-5}$.

A similar result was found with channels activated by (-)-pentobarbital. Since this anesthetic barbiturate induces current fluctuations having a corner frequency of 1.23 Hz (Mathers and Barker, 1980), the single channel currents are readily visible even at a recording bandwidth of $60 \mathrm{~Hz}$ (Fig. $3 A$ ). The narrower bandwidth is evident in the longer rise times of the channel currents in these data, but detail in the open time distribution is still discernible (Fig. $3 B$ ). Two of the four experiments provided enough channel events to construct open state lifetime distributions. In both cases, the semilog plots were clearly nonlinear, and a better fit to the open time distributions was obtained by employing a sum of two exponential functions. Thus, the continuous curves in Figures 2 and $3 B$ were calculated from an expression of the form

$$
N(t)=N_{F} e^{-t / \tau_{F}}+N_{S} e^{-t / \tau_{S}}
$$

where $\tau_{F}$ and $\tau_{S}$ denote the time constants of the "fast" and "slow" components, and $N_{F}$ and $N_{S}$ are the total number of each type of event. The four parameters, $N_{F}$, $N_{S}, \tau_{F}$, and $\tau_{S}$, were varied to obtain a least squares fit to the data. For the particular experiments shown in Figures $2, B$ and $C$, and $3 B$, the double exponential fit decreased the total RMS deviation by factors of between 5 and 10 . In contrast, the fit to the data in Figure $2 A$ was improved by only $30 \%$ in comparison with the RMS of a single exponential fit.

The parameters obtained from best fitting double exponential functions to six muscimol, two (-)-pentobarbital, and one GABA experiment are presented in Table I. In two GABA experiments, the quality of the fit was not improved significantly with a double exponential function; therefore, the parameters of a single exponential function are displayed in the table. The error estimates are derived for each individual fit and represent the reliability of each parameter. The values presented in the table show considerable variability. Some of the smaller values for $\tau_{F}$ approach the response time of the detecting system, 2 msec.

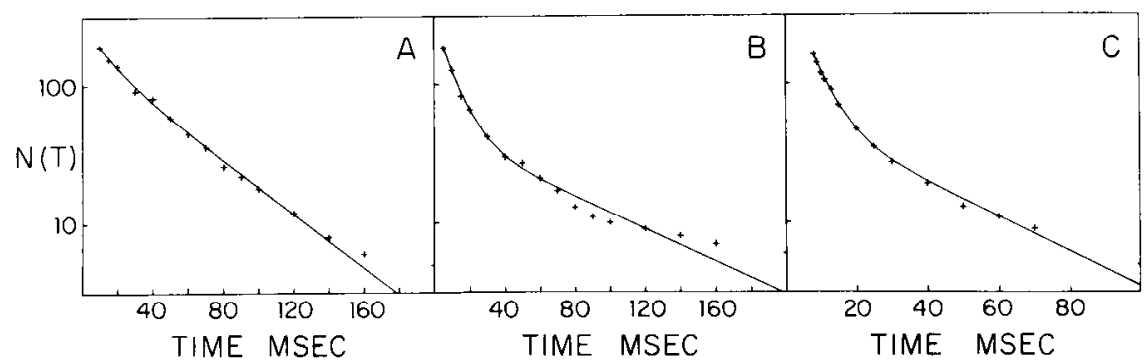

Figure 2. Semilogarithmic plots of cumulative open state lifetime distributions. The ordinate, $N(t)$, is the number of events having duration $t$ or longer. The curves are the best fitting sums of two exponential functions as discussed in the text. $A$ and $B$, Muscimol; $C$, GABA. These records represent a compilation of events from records, such as those of Figure 1, taken from a single membrane patch. Typical experiments record about 200 current jumps. 
A

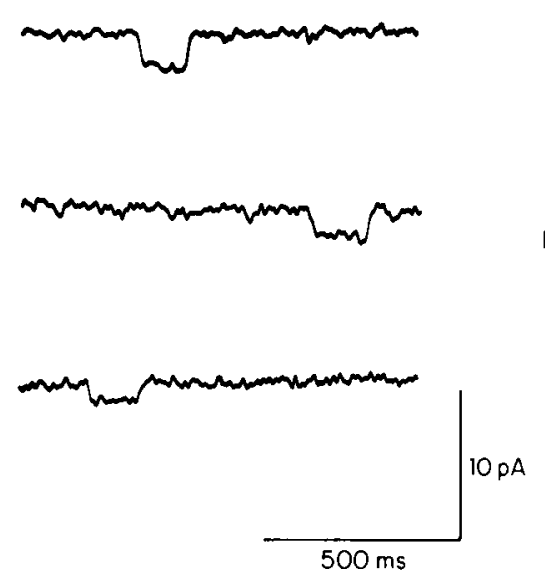

B

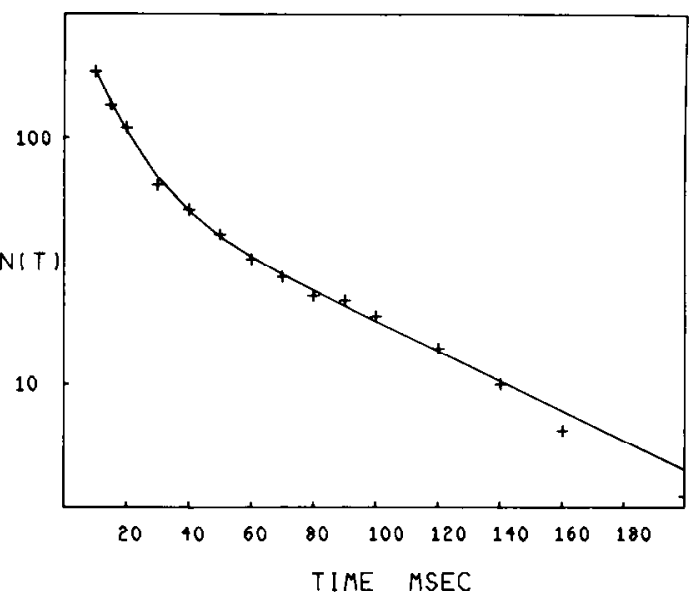

Figure 3. $A$, Current jumps induced by (-)-pentobarbital; $B$, cumulative open state lifetime distributions of channels activated by (-)-pentobarbital obtained as in Figure 2.

\section{Discussion}

The three agonists used in this study all open ionic channels in the cell membranes of spinal cord neurons. A single conducting state is seen for each compound and the unit conductance is the same in each case (Mathers and Barker, 1981b; Barker et al., 1981). The observation that agonists of the same postsynaptic receptor produce events with the same unit conductance has been noted in other systems as well (Jackson et al., 1982; Colquhoun et al., 1975).

The channel conductances measured here exceed the mean values estimated from noise analysis in the same preparation by factors varying from 1.3 to 1.9 . The consistency of this discrepancy for three different compounds suggests that it may be systematic. GABA-activated channel conductances also have been estimated from noise analysis on crayfish muscle, $9 \mathrm{pS}$ (Dudel et al., 1980), and on locust muscle, $22 \mathrm{pS}$ (Cull-Candy and Miledi, 1981). These results cannot be compared with ours because the ionic conditions in these experiments were different.

When noise is generated by low concentrations of agonist, the rate of channel opening is much slower than the rate of channel closing, and the relaxation time taken from a single Lorentzian power spectrum then reduces to the channel mean open time. The open time distributions of Figures 2, $B$ and $C$, and $3 B$ do not yield a unique mean open time. In comparing these single channel measurements with earlier noise analysis on the same preparation (McBurney and Barker, 1978; Mathers and Barker, 1980, 1981b; Barker et al., 1981), it must be borne in mind that slower processes contribute more power per unit of band frequency. The power spectral density, $W(f)$, of current fluctuations generated by two independent kinetic processes of random current pulses, both with amplitude $i$, with relative frequencies $\nu_{S}$ and $\nu_{F}$ per unit time, and mean durations $\tau_{S}$ and $\tau_{F}$, is given by the sum of two Lorentzian expressions

$$
W(f)=4 i^{2}\left\{\frac{\nu_{S} \tau_{S}^{2}}{1+\left(2 \pi \tau_{S} f\right)^{2}}+\frac{\nu_{F} \tau_{F}{ }^{2}}{1+\left(2 \pi \tau_{F} f\right)^{2}}\right\}
$$

With the ratio of the frequencies of fast and slow events, $\nu_{F} / \nu_{S}$, given by $N_{F} / N_{S}$, the spectrum of equation 2 is of the form expected from the channel open time distribution of equation 1 . Dividing equation 1 by the total time of recording and multiplying by $i^{2}$ produces an equation which is related to equation 2 by the Wiener-Khintkhine relation (at low channel frequencies, where the open state lifetime distribution approximates the autocorrelation function). Note that integration of equation 2 yields a total noise variance of $i^{2}\left(\nu_{S} \tau_{S}+\nu_{F} \tau_{F}\right)$ as expected.

Equation 2 illustrates how the square of the relaxation time determines the relative contribution to the spectral density of two independent processes. This expression can be used to simulate noise spectra which would result from channels with two different closing rates; these rates can be taken from the parameters of double exponential curve fits. Spectra simulated in this fashion are dominated by the slow kinetic process and can therefore be fit by a single Lorentzian term with a time constant close to $\tau_{\mathrm{s}}$

In the analysis of muscimol-induced noise, observed spectra appear to be dominated by this slow kinetic process (Mathers and Barker, 1980, 1981b; Barker et al., 1981). Mean open state lifetimes inferred from noise can be predicted by equation 2 . The general formula obtained by solving equation 2 for the value of $f$ for which $W(f)$ $=W(0) / 2$ is quite complicated, but for values of $N_{F} / N_{S}$, $\tau_{F}$, and $\tau_{S}$ given for muscimol or (-)-pentobarbital in Table I, this would reduce to $\tau_{\text {noise }}=\tau_{S}$. Thus, the value to which the noise-derived relaxation time should be compared is just the mean duration of the slow component, $\tau_{S}$. These numbers are in fair quantitative agreement, reproducing the sequence of time constants in decreasing order: (-)-pentobarbital, muscimol, and GABA (Mathers and Barker, 1980, 1981b).

Single channel analysis reveals an additional faster process which would be difficult to detect in a power spectrum. If the parameters of the second muscimol experiment of Table I are used to calculate the relative contributions of the two Lorentzian terms of equation 2 , it is seen that, at low frequencies, the slow process 
generates 11 times as much noise as the fast process. Although the value of $N_{F} / N_{S}$ indicates that there are 5.3 times as many fast events, as is evident in Figure $2 B$, the $\tau^{2}$ weighting of the noise power per unit of band frequency is an over-riding effect. Selected noise experiments indicate that this faster process can be seen in power spectra (Mathers and Barker, 1981a) provided that the proportion of fast events is sufficiently high.

A faster process that is partially lost in the spectral analysis of channel noise would contribute to the mean current but not to its variance, regardless of whether variance is measured directly or from the integral of the best fitting single Lorentzian power spectrum. Since the single channel conductance is obtained from the ratio of the variance to the mean, estimates of the channel conductance from the analysis of noise induced by GABA agonists, or in other preparations with a similar combination of fast and slow fluctuations, would be low. A loss of some of the high frequency noise, or other factors related to differences in driving force resulting from impalement with two microelectrodes instead of one, could account for the systematic discrepancy noted earlier between single channel and noise measurements of unit conductance.

The significance of this faster process is as yet unclear. Analogous fast processes have been observed in the closing of acetylcholine receptor channels in tissue-cultured muscle from rat (M. B. Jackson, C. Morris, B. Wong, H. Lecar, and C. N. Christian, submitted for publication) and from human beings (Jackson et al., 1981). The existence of two components in the kinetics of the gating of chemically activated channels also has been inferred from studies of rat submandibular ganglion cells (Rang, 1981 ) and the snake neuromuscular junction (Dionne and Parsons, 1981).

The open state lifetime distribution is a reflection of the underlying mechanism of the channel closing transition. The deviation of distributions from a single exponential shape indicates that the simplest model of a single population closing by a first order process can be eliminated. Open state lifetime distributions which are not single exponential can be generated by models of channel gating which include transitions between two or more different open states of the channel (Colquhoun and Hawkes, 1981). Two different populations of independently gated channels also would produce lifetime distributions of the form reported here.

Given the rank order of mean open state lifetimes for GABA, muscimol, and (-)-pentobarbital (Mathers and Barker, 1980, 1981b), clear resolution of faster processes in GABA channel kinetics is more difficult. Only one of the three GABA experiments of Table I-an experiment with an especially good time response and low noise level-resulted in an open time distribution with marked deviation from a single exponential shape (Fig. $2 C$; Table I). This raises the possibility that a faster process operates in the closing of GABA-activated channels as well but is often lost upon filtering the signal.

A survey of the values of the parameters derived from muscimol in Table I reveals that there is variability between different experiments. Note that the standard deviations of $\bar{\tau}_{S}$ and $\bar{\tau}_{F}$ (Table I) exceed the uncertainties for these parameters estimated from the uniqueness of each individual curve fit. Variability in the kinetics of channel closing is difficult to characterize without large numbers of experiments. The variability seen here is comparable in scale to the variability found in a more extensive study of neutral amino acid-induced current fluctuations in spinal neurons (Barker et al., 1981). In the six muscimol experiments of Table I, it is impossible to distinguish a clear pattern. Variability in channel closing kinetics also was seen in studies of the acetylcholine receptor channel from human muscle (Jackson et al., 1982). In this case, the variability could be explained in terms of variations in the ratio $N_{S} / N_{F}$, with $\tau_{F}$ and $\tau_{S}$ relatively constant.

These experiments indicate that GABA, muscimol, and (-)-pentobarbital activate ionic channels in central neuronal membranes in a manner similar to that reported for transmitters acting on membranes peripheral to the CNS. The single channel observations show that the actions of the putative neurotransmitter GABA are mimicked by compounds as diverse as a plant alkaloid and a clinically important anesthetic. The application of the patch electrode technique to cultured mammalian spinal cord supports the view advanced by noise analysis that ionic channels are responsible for the inhibitory responses elicited by GABA and other agents. These measurements reveal additional complexity in the kinetic behavior of these channels which was not evident in the previous analysis of conductance fluctuations. The ultimate elucidation of the mechanism of channel gating will contribute to our understanding of synaptic physiology and will generate a helpful framework for the further investigation of drugs which alter inhibitory synaptic transmission at the postsynaptic level.

Addendum. Shortly after this paper was submitted, Colquhoun and Sakmann (1981) reported a very rapid component in the kinetics of the closing of acetylcholine receptor channels and distributions of open times which appeared to be sums of two exponential functions.

\section{References}

Anderson, C. R., and C. F. Stevens (1973) Voltage clamp analysis of acetylcholine produced end-plate current fluctuations at frog neuromuscular junction. J. Physiol. (Lond.) 235: $655-691$.

Barker, J. L., and B. R. Ransom (1978) Amino acid pharmacology of mammalian central neurones grown in tissue culture. J. Physiol. (Lond.) 280: 331-354.

Barker, J. L., R. N. McBurney, and J. F. MacDonald (1981) Fluctuation analysis of neutral amino acid responses in cultured mouse spinal neurones. J. Physiol. (Lond.), in press.

Colquhoun, D., and A. G. Hawkes (1981) On the stochastic properties of single ion channels. Proc. R. Soc. Lond. (Biol.) 211: 205-235.

Colquhoun, D., and B. Sakmann (1981) Fluctuations in the microsecond time range of the current through single acetylcholine receptor ion channels. Nature 294: 464-466.

Colquhoun, D., V. E. Dionne, J. H. Steinbach, and C. F. Stevens (1975) Conductance of channels opened by acetylcholine-like drugs in muscle end-plate. Nature 253: 204-206.

Cull-Candy, S. G., and R. Miledi (1981) Junctional and extrajunctional membrane channels activated by GABA in locust muscle fibres. Proc. R. Soc. Lond. (Biol.) 211: 527-535.

Dionne, V. E., and R. L. Parsons (1981) Characteristics of the 
acetylcholine-operated channel at twitch and slow fibre neuromuscular junctions of the garter snake. J. Physiol. (Lond.) 310: 145-158.

Dudel, J., W. Finger, and H. Stettmeier (1980) Inhibitory synaptic channels activated by $\gamma$-aminobutyric acid (GABA) in crayfish muscle. Pfluegers Arch. 387: 143-151.

Hamill, O. P., A. Marty, F. Neher, B. Sakmann, and F. J. Sigworth (1981) Improved patch-clamp techniques for highresolution current recording from cells and cell-free membrane patches. Pfluegers Arch. 391: 85-100.

Jackson, M. B., and H. Lecar (1979) Single post-synaptic channel currents in tissue cultured muscle. Nature 282: 863-864.

Jackson, M. B., H. Lecar, V. Askanas, and W. K. Engel (1982) Single cholinergic receptor channel currents in cultured human muscle. J. Neurosci. 2: in press.

Katz, B., and R. Miledi (1970) Membrane noise produced by acetylcholine. Nature 226: 962-963.

Mathers, D. A., and J. L. Barker (1980) (-)Pentobarbital opens ion channels of long duration in cultured mouse spinal neurons. Science 209: 507-509.

Mathers, D. A., and J. L. Barker (1981a) Low agonist concentrations reveal kinetic behavior of GABA-induced membrane channels in tissue cultured mouse spinal neurons. Soc. Neurosci. Abstr. 7: 726.

Mathers, D. A., and J. L. Barker (1981b) GABA and muscimol open ion channels of different lifetimes on cultured mouse spinal cord cells. Brain Res. 204: 242-247.

Mathers, D. A., M. B. Jackson, H. Lecar, and J. L. Barker (1981) Single channel currents activated by GABA, muscimol, and (-)pentobarbital in cultured mouse spinal neurons. Biophys. J. 33: 14a.

McBurney, R. N., and J. L. Barker (1978) GABA-induced conductance fluctuations in cultured spinal neurones. Nature 274: 596-597.

Neher, E., and B. Sakmann (1976) Single-channel currents recorded from membrane of denervated frog muscle fibres. Nature 260: 799-802.

Nelson, D. J., and F. Sachs (1979) Single ionic channels observed in tissue-cultured muscle. Nature 282: 861-863.

Rang, H. P. (1981) The characteristics of synaptic currents and responses to acetylcholine of rat submandibular ganglion cells. J. Physiol. (Lond.) 311: 23-55.

Ransom, B. R., E. Neale, M. Henkart, M. Bullock, and P. G. Nelson (1977a) Mouse spinal cord in cell culture. I. Morphology and intrinsic neuronal electrophysiologic properties. J. Neurophysiol. 40: 1132-1150.

Ransom, B. R., P. N. Bullock, and P. G. Nelson (1977b) Mouse spinal cord in cell culture. III. Neuronal chemosensitivity and its relation to synaptic activity. J. Neurophysiol. 40: 11631177.

Sachs, F., and H. Lecar (1973) Acetylcholine noise in tissue cultured muscle cells. Nature New Biol. 246: 214-216. 